ARTICLE

https://doi.org/10.1038/s41467-019-13791-1

\title{
Ultrafast transient liquid assisted growth of high current density superconducting films
}

\author{
L. Soler (10 1, J. Jareño1, J. Banchewski', S. Rasi (i) ${ }^{2,1}$, N. Chamorro (i) ${ }^{3,1}$, R. Guzman (1) 1, R. Yáñez (1) ${ }^{3}$, \\ C. Mocuta (1) ${ }^{4}$, S. Ricart ${ }^{1}$, J. Farjas (1) ${ }^{2}$, P. Roura-Grabulosa ${ }^{2}$, X. Obradors ${ }^{1} \&$ T. Puig (i) ${ }^{1 \star}$
}

The achievement of high growth rates in $\mathrm{YBa}_{2} \mathrm{Cu}_{3} \mathrm{O}_{7}$ epitaxial high-temperature superconducting films has become strategic to enable high-throughput manufacturing of long length coated conductors for energy and large magnet applications. We report on a transient liquid assisted growth process capable of achieving ultrafast growth rates $\left(100 \mathrm{~nm} \mathrm{~s}^{-1}\right)$ and high critical current densities ( $5 \mathrm{MA} \mathrm{cm}^{-2}$ at $77 \mathrm{~K}$ ). This is based on the kinetic preference of $\mathrm{Ba}-\mathrm{Cu}-\mathrm{O}$ to form transient liquids prior to crystalline thermodynamic equilibrium phases, and as such is a non-equilibrium approach. The transient liquid-assisted growth process is combined with chemical solution deposition, proposing a scalable method for superconducting tapes manufacturing. Additionally, using colloidal solutions, the growth process is extended towards fabrication of nanocomposite films for enhanced superconducting properties at high magnetic fields. Fast acquisition in situ synchrotron X-ray diffraction and high resolution scanning transmission electron microscopy (STEM) become crucial measurements in disentangling key aspects of the growth process.

\footnotetext{
${ }^{1}$ Institut de Ciència de Materials de Barcelona, ICMAB-CSIC, Campus UAB, 08193 Bellaterra, Catalonia, Spain. ${ }^{2}$ GRMT, Department of Physics, Universitat de Girona, Campus Montilivi, Edif. PII, E17003 Girona, Catalonia, Spain. ${ }^{3}$ Departament de Química, Universitat Autònoma de Barcelona, 08193 Bellaterra, Catalonia, Spain. ${ }^{4}$ Synchrotron SOLEIL, L'Orme des Merisiers Saint-Aubin BP 48, 91192 Gif-sur-Yvette, France. *email: teresa.puig@icmab.es
} 
$\mathrm{T}$ he discovery of cuprate superconductors, 30 years ago, raised enormous hope for widespread use of superconductivity in many large-scale energy applications and powerful magnets. However, huge challenges needed to be addressed to provide a flexible conductor with high current transport capabilities. Coated conductors (CCs) revolutionized this area by growing epitaxial $\mathrm{REBa}_{2} \mathrm{Cu}_{3} \mathrm{O}_{7}(\mathrm{REBCO}, \mathrm{RE}=$ Rare Earth or $\mathrm{Y}$ ) layers on top of buffered long length flexible metallic substrates. At present, they are the materials with highest current capabilities and the most promising to extend large-scale applications of superconductivity beyond those achieved with lowtemperature superconductors ${ }^{1-3}$. Remarkable relevance was achieved by the growth of nanocomposites ${ }^{4-7}$, which strongly enhanced performances at high magnetic fields. However, the low throughput in the production of CCs remains a severe limitation to generate drastic cost reductions of CCs for wide market implementation.

Chemical solution deposition (CSD) is a non-vacuum approach leading to CCs with very competitive performance, while having a strong potential for achieving the above-mentioned cost reductions. Nevertheless, the low throughput owing to small growth rates $\left(\sim 1 \mathrm{~nm} \mathrm{~s}^{-1}\right)$ from the most extended trifluoracetateroute $(\text { TFA })^{8}$ still restricts from full development of CSD potentialities.

The approach identified to reach high film growth rates, is that based on liquid-assisted growth ${ }^{9-11}$. In this case, fast atomic diffusion and high atomic density encompass a much higher growth rate than in vapor or solid diffusion reaction approaches $^{12}$. More recently, few examples of liquid-assisted growth of REBCO films are found, all based on vacuum deposition techniques ${ }^{10,11,13-16}$.

Here, we report on an outstanding process that goes beyond the established schemes based on growth from liquids appearing in the equilibrium phase diagram. It combines CSD using nonfluorinated precursors and a non-equilibrium process grounded on transient liquids, originating an ultrafast growth approach: Transient Liquid-Assisted Growth based on CSD (TLAG-CSD). The kinetic hindrances in crystallization are advantageously used to induce transient liquids, i.e., liquids that do not exist in the equilibrium ternary phase diagram for such compositions. This idea results in growth rates of $\sim 100 \mathrm{~nm} \mathrm{~s}^{-1}$ for epitaxial YBCO films, as demonstrated in this work by in situ synchrotron X-ray diffraction studies. In addition, the $T-P_{\mathrm{O} 2}$ window for heteroepitaxial growth is strongly enlarged and the process is shown to be compatible with scalable solution deposition techniques. We further prove that TLAG-CSD nanocomposite films, with high critical temperatures and current densities, can be grown with the above depicted approach using colloidal solutions of $5 \mathrm{~nm}$-size $\mathrm{BaMO}_{3}(\mathrm{M}=\mathrm{Zr}$, Hf $)$ preformed nanoparticles. These nanocomposites display relevant nanostructural changes that positively influence vortex pinning at high magnetic fields. The synergistic effect between nanoparticles and local nanoscale lattice distortions (nanostrain) observed, brings additional opportunities to further improved vortex pinning efficiency of TLAG-CSD nanocomposites. In addition, TLAG-CSD brings opportunities for simplified reactors and precursors flexibility, thus it is compliant with a high-throughput method for easier market penetration.

\section{Results}

TLAG principles. The TLAG process is based on the capability of the ternary system $\mathrm{BaCuO}_{2}, \mathrm{CuO}$, and $\mathrm{Y}_{2} \mathrm{O}_{3}$ to achieve ultrafast growth of YBCO films. This is possible because the eutectic reaction between the first two compounds is able to form a transient liquid (with the presence of dispersed $\mathrm{Y}_{2} \mathrm{O}_{3}$ solid nanoparticles) in the region of the phase diagram where solid $\mathrm{YBa}_{2} \mathrm{Cu}_{3} \mathrm{O}_{7-\mathrm{x}}$ is the equilibrium phase (light gray region of Fig. 1b). The idea is that there is an energy barrier for nucleation of the crystalline phase (which delays its formation through an incubation time), whereas the transient liquid phase can emerge instantaneously, thus enabling the development of an ultrafast non-equilibrium processing approach ${ }^{17}$.

Upon dissolution of the $\mathrm{Y}_{2} \mathrm{O}_{3}$ nanoparticles in the $\mathrm{Ba}-\mathrm{Cu}-\mathrm{O}$ transient liquid and $\mathrm{Y}$ diffusion toward the substrate interface (see diagram of Fig. 1a), epitaxial growth of YBCO occurs. The a
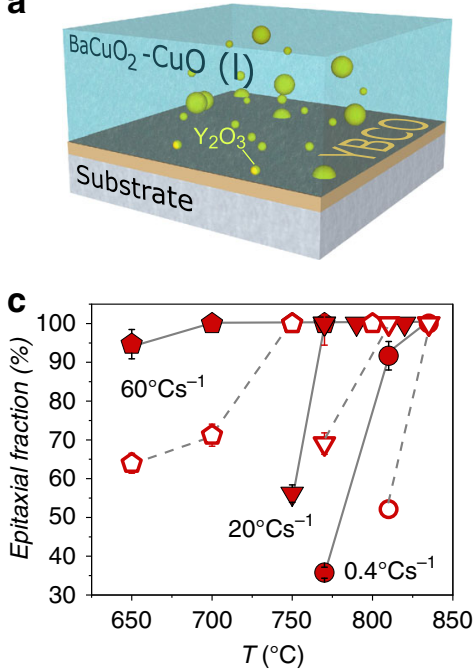

b

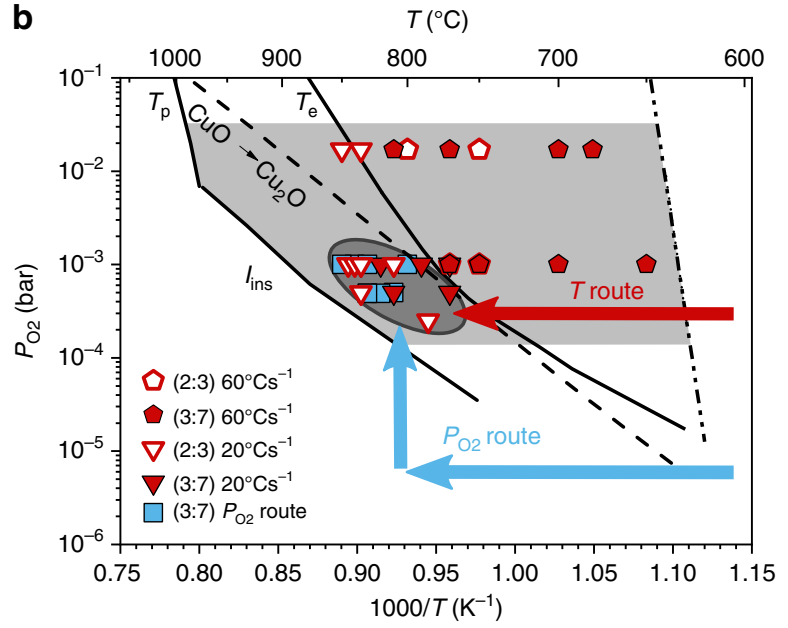

Fig. 1 Transient liquid-assisted growth (TLAG) principles. a Schematic drawing of the TLAG process. $\mathbf{b} T-P_{\mathrm{O} 2}$ phase diagram for YBCO TLAG growth. The $2 \mathrm{BaCuO}_{2}-\mathrm{CuO}$ eutectic temperature, $T_{\mathrm{e}}$, the $\mathrm{YBCO}$ peritectic temperature, $T_{\mathrm{p}}$, and the instability $\mathrm{YBCO}$ line, $l_{\text {ins }}$ are shown. The T-route and $\mathrm{P}_{\mathrm{O} 2}$-route are indicated by arrows. Symbols are the final temperature and $P_{\mathrm{O} 2}$ values of individual experiments: open symbols $\left(2 \mathrm{BaCuO}_{2}-\mathrm{CuO}_{1} 2: 3\right.$ composition) or closed symbols ( $3 \mathrm{BaCuO}_{2}-4 \mathrm{CuO}, 3: 7$ composition) in red ( $\mathrm{T}$-route) or blue ( $\mathrm{P}_{\mathrm{O} 2}$-route); all symbols indicate $100 \%$ epitaxial growth. $T_{\mathrm{e}}$ has been determined from ref. ${ }^{27}$ and this article (see details in methods); $T_{p}, T_{\text {ins, }}$ and the $\mathrm{CuO}-\mathrm{Cu}_{2} \mathrm{O}$ line are from ref. ${ }^{10}$. $\mathrm{c}$ Epitaxial fraction dependence on growth temperature for the T-route at different heating rates for $2: 3$ (empty symbols) and 3:7 (solid symbols) compositions at $P_{\mathrm{O} 2}=10^{-3}$ bar. Error bars indicate the accuracy of the method as determined in ref. ${ }^{7}$. 
a

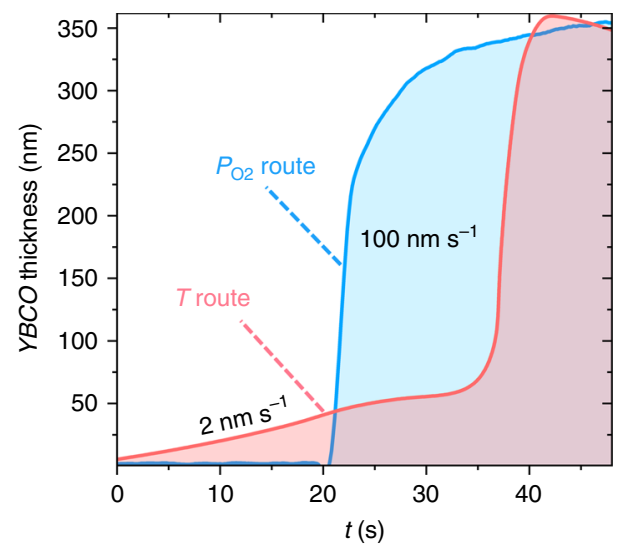

b
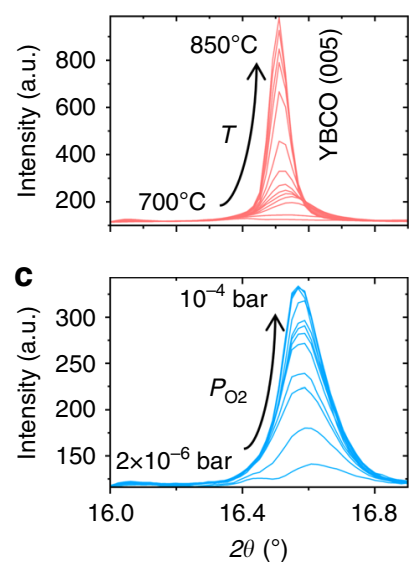

d
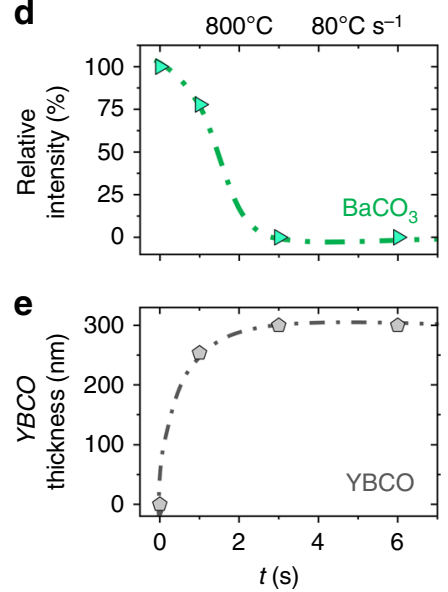

Fig. 2 Fast evolution of phases in TLAG by in situ XRD synchrotron radiation. a YBCO growth rate determined from in situ XRD synchrotron analysis of a $350 \mathrm{~nm}$ film demonstrating $100 \mathrm{~nm} \mathrm{~s}^{-1}$ growth rate for T-route $\left(P_{\mathrm{O} 2}=10^{-3}\right.$ bar, $\left.d T / d t=5^{\circ} \mathrm{C} \mathrm{s}^{-1}\right)$ and $P_{\mathrm{O} 2}-$ route $\left(T=850^{\circ} \mathrm{C}, P_{\mathrm{O} 2 \text {, base }}=10^{-5}\right.$ bar, $P_{\mathrm{O} \text {, final }}=10^{-2}$ bar). Initial stages for the T-route show a growth rate of $2 \mathrm{~nm} \mathrm{~s}^{-1}$. b, c Evolution of the diffracted intensity of the (005) YBCO peak for the $\mathrm{T}$-route and $\mathrm{P}_{\mathrm{O} 2}$-route, respectively, during the transformation for the experiment reported in $\mathbf{a}$. The $\mathrm{X}$-ray energy used for these measurements was $18 \mathrm{keV}$. The evolution with time of the XRD intensity for $\mathbf{d ~} \mathrm{BaCO}_{3}$ and $\mathbf{e} \mathrm{YBCO}$ phases in a T-route process in a $\mathrm{RTA}$ furnace at $800^{\circ} \mathrm{C}\left(P_{\mathrm{O} 2}=10^{-2}\right.$ bar, $d T / d t=80^{\circ} \mathrm{C} \mathrm{s}^{-1}$ ) for a film of $300 \mathrm{~nm}$. Notice that $\mathrm{BaCO}_{3}$ consumption to form the transient liquid goes in parallel with $\mathrm{YBCO}$ growth, which reaches completion in $3 \mathrm{~s}$ in these conditions.

fast $\mathrm{Y}$ diffusion in the liquid may boost the growth rate. For instance, the diffusivity of $\mathrm{Y}$ at $970{ }^{\circ} \mathrm{C}$ in the $\mathrm{BaCuO}_{2}-\mathrm{CuO}$ liquid is $4 \times 10^{-10} \mathrm{~m}^{2} \mathrm{~s}^{-118}$, whereas in the YBCO solid is almost two decades lower, of only $8 \times 10^{-12} \mathrm{~m}^{2} \mathrm{~s}^{-119}$. This makes liquidassisted processes potentially so much faster than solid-solid or solid-gas reactions. We anticipate that the crystallization through the liquid will be mostly limited by the substrate interfacial kinetics of $\mathrm{Y}, \mathrm{Ba}, \mathrm{Cu}$, and $\mathrm{O}$ atoms ${ }^{20,21}$.

Our results demonstrate that crystallization rates of at least $100 \mathrm{~nm} \mathrm{~s}^{-1}$ are reachable. In addition, we show that the liquid can be formed below the binary $\mathrm{BaCuO}_{2}-\mathrm{CuO}$ equilibrium eutectic temperature, $T_{\mathrm{e}}$ (Fig. 1b), thus TLAG-YBCO epitaxial growth is achieved in a wide region of the phase diagram (light gray region of Fig. 1b). In summary, the kinetic crystallization hindrances are exploited to induce much faster crystallization through a transient liquid.

In general, epitaxial film growth is ensured by properly tuning the supersaturation conditions. In the TLAG case, liquid composition $^{11,22}$ and $\mathrm{RE}$ element solubility in the melt ${ }^{23}$ are the most relevant parameters. Several process parameters (temperature, oxygen partial pressure $P_{\mathrm{O} 2}$, total pressure of gases in the reactor $P_{\text {total }}$, heating ramp rates) have to be optimized for epitaxial growth for each composition. In this work, two different liquid compositions were studied, $2 \mathrm{BaCuO}_{2}-\mathrm{CuO}(2: 3)$ and $3 \mathrm{BaCuO}_{2}-4 \mathrm{CuO}$ (3:7), which correspond to the $\mathrm{YBa}_{2} \mathrm{Cu}_{3} \mathrm{O}_{\mathrm{x}}$ and $\mathrm{YBa}_{2} \mathrm{Cu}_{4.66} \mathrm{O}_{\mathrm{x}}$ film compositions, respectively. The latter matches the $\mathrm{Ba}-\mathrm{Cu}-\mathrm{O}$ eutectic liquid composition and results in the desired phase $\left(\mathrm{YBa}_{2} \mathrm{Cu}_{3} \mathrm{O}_{\mathrm{x}}\right)$ together with $\mathrm{CuO}$ nanoparticles, mainly observed at the film surface. Both melts give rise to epitaxial YBCO films.

Our results reveal that the temperature window for $c$ axis epitaxy is widened to temperatures below $T_{\mathrm{e}}$ and that this expansion depends on the heating ramp (down to $650{ }^{\circ} \mathrm{C}$ for the $3: 7$ composition at $d T / d t=60^{\circ} \mathrm{C} / \mathrm{s}$, Fig. $1 \mathrm{~b}, \mathrm{c}$ ). In fact, as TLAG is a non-equilibrium kinetically driven process, when high heating rates are used, the $\mathrm{Y}$ ions dissolution from the $\mathrm{Y}_{2} \mathrm{O}_{3}$ nanoparticles into the melt might be compromised, and the $\mathrm{Y}$ concentration in the liquid, $C_{Y}$, will decrease. As a consequence, the relative supersaturation of the liquid, $\sigma=\left(C_{\mathrm{Y}} / C_{\mathrm{e}}-1\right.$ ) (where $C_{\mathrm{e}}$ is the equilibrium concentration), will decrease, leading to the enhanced $c$ axis nucleation and growth window observed. This fact brings unique opportunities for fine tuning of the film manufacturing process by employing rapid thermal annealing furnaces.

When the two liquids are compared, one realizes that the 3:7 eutectic composition is able to trigger the formation of $c$ axis YBCO at lower temperatures (solid versus open symbols of Fig. 1b, c). This behavior is in agreement with a lower supersaturation for the 3:7 liquid composition. If $C_{\mathrm{Y}}$ is assumed similar for both liquids, since $C_{\mathrm{e}}$ increases with $\mathrm{Cu}$ content in the liquid $^{24}$, supersaturation will indeed be lower for the 3:7 liquid composition $^{25}$.

The best conditions achieved so far, demonstrating high critical current density in the superconducting films, are shown in Fig. 1b as the dark gray encircled region (for both compositions and different TLAG routes investigated in this work, see details below). It is worth noting that reaching this high-temperature region in a very short time lapse is very important because here is where the growth rate is larger: ultrahigh values in the range of $100 \mathrm{~nm} \mathrm{~s}^{-1}$ have been demonstrated for $T>T_{\mathrm{e}}$, whereas two orders of magnitude lower growth rates $\left(2 \mathrm{~nm} \mathrm{~s}^{-1}\right)$ are reached at lower temperatures (see Fig. 2a). This is further confirmed in Fig. 2e, where the fast growth of TLAG is obtained from rapid thermal annealed samples quenched at $800^{\circ} \mathrm{C}$, demonstrating that $3 \mathrm{~s}$ are enough to grow a $300 \mathrm{~nm}$ YBCO film (i.e., $100 \mathrm{~nm} \mathrm{~s}^{-1}$ ) with a heating ramp of $80^{\circ} \mathrm{Cs}^{-1}$.

TLAG-CSD approach. The potential for high-throughput and low-cost TLAG-CSD CCs is based on the idea of combining the ultrafast growth of TLAG with CSD. A stable and atomically homogeneous solution of $\mathrm{Y}, \mathrm{Ba}$ and $\mathrm{Cu}$ salts based on propionate precursors is deposited on a substrate and pyrolyzed to reach a homogeneous nanoporous layer of $\mathrm{Y}_{2} \mathrm{O}_{3}, \mathrm{BaCO}_{3}$ and $\mathrm{CuO}$ nanocrystalline phases (details given in Methods). Then, the sample is heated up to the gray region of Fig. 1b, where upon $\mathrm{BaCO}_{3}$ reaction, a homogeneous dispersion of $\mathrm{Y}_{2} \mathrm{O}_{3}(\mathrm{~s})$ nanoparticles is formed in the $\mathrm{BaCuO}_{2}-\mathrm{CuO}$ (liquid), to crystallize into epitaxial YBCO.

The TLAG-CSD process is compatible with multideposition of pyrolyzed layers and scalable ink jet printing patterning 
techniques, where layer thickness is not only controlled by the solution concentration but also by drop volume and drop spacing ${ }^{26}$. Pyrolyzed layers up to $1 \mu \mathrm{m}$ thick deposited by ink jet printing show a high degree of homogeneity throughout thickness (see Supplementary Figure 1a). These layers are transformed after the TLAG growth process to homogeneous, pore free, and epitaxial YBCO films of $550 \mathrm{~nm}$ (Supplementary Figure $1 b$ ), where straight twin boundaries crossing the total film thickness can be identified. Higher magnification with atomic resolution scanning transmission electron microscopy (STEM) confirms the highly crystalline quality of the epitaxial growth.

The versatility and speed of the TLAG-CSD growth process has been further investigated by in situ fast acquisition $\mathrm{x}$-ray diffraction analysis at SOLEIL synchrotron (Fig. 2 and Supplementary Figure 2) and advanced electron transmission microscopy from quenched samples (Supplementary Figure 3). Both techniques enabled us to disentangle the phase transformations and growth mechanisms. A key point to understand the TLAGCSD growth mechanism was the identification of the reaction of $\mathrm{BaCO}_{3}$ with $\mathrm{Cu}$-phases to form the $\mathrm{Ba}-\mathrm{Cu}-\mathrm{O}$ intermediate phases.

We have investigated two different routes indicated in Fig. 1b with arrows, temperature route (T-route, with red arrows) and oxygen partial pressure route $\left(\mathrm{P}_{\mathrm{O} 2}\right.$-route, with blue arrow). In the T-route, $\mathrm{BaCO}_{3}$ reacts with $\mathrm{CuO}$ during heating at a fixed oxygen partial pressure $\left(10^{-4} \mathrm{bar}<P_{\mathrm{O} 2}<10^{-2} \mathrm{bar}\right)$; then the transient liquid forms dissolving $\mathrm{Y}$ ions from $\mathrm{Y}_{2} \mathrm{O}_{3}$ to give rise to $\mathrm{YBCO}$ (see in situ X-ray powder diffraction (XRD) synchrotron studies reported in Supplementary Figure 2). This reaction process is represented in Eq. (1) (not adjusted since we have used two considerably different liquid compositions: $2 \mathrm{BaCuO}_{2}-\mathrm{CuO}(2: 3)$ and the $\left.3 \mathrm{BaCuO}_{2}-4 \mathrm{CuO}(3: 7)\right)$.

$$
\begin{aligned}
\mathrm{BaCO}_{3}(\mathrm{~s}) & +\mathrm{CuO}(\mathrm{s})+\mathrm{Y}_{2} \mathrm{O}_{3}(\mathrm{~s}) \rightarrow[\mathrm{Ba}-\mathrm{Cu}-\mathrm{O}](\mathrm{l})+\mathrm{Y}_{2} \mathrm{O}_{3}(\mathrm{~s}) \\
& \rightarrow \mathrm{YBa}_{2} \mathrm{Cu}_{3} \mathrm{O}_{6.5}(\mathrm{~s})+\mathrm{xCuO}(\mathrm{s})
\end{aligned}
$$

where the labels $(s),(l)$ stand for solid and liquid, respectively, and $x=0$ is for (2:3) and $x \neq 0$ for (3:7) compositions used.

In the $\mathrm{P}_{\mathrm{O} 2}$-route (blue arrows of Fig. $1 \mathrm{~b}$ ), $\mathrm{BaCO}_{3}$ reacts during heating at low (and constant) oxygen partial pressure $\left(10^{-5}\right.$ bar $<$ $P_{\mathrm{O} 2 \text {,base }}<10^{-4}$ bar) in the region where $\mathrm{YBCO}$ is not the stable phase (i.e., below the instability line, $I_{\text {ins }}$, of Fig. $1 \mathrm{~b}$ ), following the reaction process shown in Eq. (2). Then, a steep increase in $P_{\mathrm{O} 2}$ $\left(10^{-4}\right.$ bar $<P_{\mathrm{O} 2 \text {,final }}<10^{-2}$ bar $)$ at constant temperature, brings the film to the right region of $T-P_{\mathrm{O} 2}$ in order to form the transient $\mathrm{Ba}-\mathrm{Cu}-\mathrm{O}$ liquid that allows fast epitaxial growth of YBCO (Eq. (3)). Hence, in this route, the elimination of $\mathrm{BaCO}_{3}$ is fully decoupled from the YBCO growth.

According to Eq. (2), initial $\mathrm{BaCO}_{3}$ and $\mathrm{Cu}_{2} \mathrm{O}$ react to generate $\mathrm{BaCu}_{2} \mathrm{O}_{2}$, which is the stable solid $\mathrm{Ba}-\mathrm{Cu}$ phase at this low $P_{\mathrm{O} 2}{ }^{27,28}$, (Supplementary Figure 2c).

$$
\begin{aligned}
\mathrm{BaCO}_{3}(\mathrm{~s}) & +\mathrm{CuO}(\mathrm{s})+\mathrm{Y}_{2} \mathrm{O}_{3}(\mathrm{~s}) \rightarrow \mathrm{BaCO}_{3}(\mathrm{~s})+\mathrm{Cu}_{2} \mathrm{O}(\mathrm{s})+\mathrm{Y}_{2} \mathrm{O}_{3}(\mathrm{~s}) \\
& \rightarrow \mathrm{BaCu}_{2} \mathrm{O}_{2}(\mathrm{~s})+\mathrm{Y}_{2} \mathrm{O}_{3}(\mathrm{~s})
\end{aligned}
$$

After the steep increase in oxygen partial pressure $\left(P_{\mathrm{O} 2}=P_{\mathrm{O} 2 \text {,final }}\right)$ at constant temperature, the $\mathrm{BaCu}_{2} \mathrm{O}_{2}$ solid phase transform to $\mathrm{Ba}-\mathrm{Cu}-\mathrm{O}$ liquid and subsequent $\mathrm{YBCO}$ growth (Supplementary Figure 2d), following the reaction process given in Eq. (3). Again Eq. $(2,3)$ are not adjusted to generalize in the liquid compositions employed. Notice that, for the mechanism detailed in Eq. $(2,3), P_{\mathrm{O} 2}$ is the crucial parameter, whereas $P_{\text {total }}$ was kept between $10^{-5}$ and $10^{-2}$ bar.

$$
\begin{aligned}
\mathrm{BaCu}_{2} \mathrm{O}_{2}(\mathrm{~s}) & +\mathrm{Y}_{2} \mathrm{O}_{3}(\mathrm{~s}) \rightarrow[\mathrm{Ba}-\mathrm{Cu}-\mathrm{O}](\mathrm{l})+\mathrm{Y}_{2} \mathrm{O}_{3}(\mathrm{~s}) \\
& \rightarrow \mathrm{YBa}_{2} \mathrm{Cu}_{3} \mathrm{O}_{6.5}(\mathrm{~s})+\mathrm{xCuO}(\mathrm{s})
\end{aligned}
$$

where $x=0$ stands for (2:3) and $x \neq 0$ for (3:7) liquid composition.

In this $\mathrm{P}_{\mathrm{O} 2}$-route case, ultrafast growth rates of $100 \mathrm{~nm} \mathrm{~s}^{-1}$ have also been reached (Fig. 2a) by controlling the steepness of the $P_{\mathrm{O} 2}$ change. Direct proof of the transient liquid phase for both, $\mathrm{T}$ - and $\mathrm{P}_{\mathrm{O} 2}$-routes, is given by the results of in situ XRD experiment reported in Supplementary Figure 2) where the crystalline precursor phases disappear at the onset of YBCO growth.

A detailed TEM analysis of the TLAG process for the T-route approach is shown in Supplementary Figure 3, where the stages of the process with the different reaction phases and their distribution are presented. The initial nanocrystalline phases observed after the pyrolysis $\left(\mathrm{BaCO}_{3}, \mathrm{CuO}\right.$, and $\left.\mathrm{Y}_{2} \mathrm{O}_{3}\right)$ are of tens of nanometers in size, so fast reaction paths are rendered possible. The $\mathrm{CuO}$ nanoparticles, of size in the range of $10-30 \mathrm{~nm}$, are distributed within a $\mathrm{BaCO}_{3}$ matrix. At an intermediate temperature step, the first heterogeneous nuclei of YBCO grow at the substrate interface whereas $\mathrm{BaCO}_{3}$ grains (size $\sim 40-50 \mathrm{~nm}$ ) remain in the bulk of the film. This confirms that the slowest reaction is the $\mathrm{BaCO}_{3}$ elimination. At the final stage, the YBCO epitaxial layer is observed and $\mathrm{BaCO}_{3}$ has completely disappeared. The $\mathrm{BaCO}_{3}$ reaction time is a key point in this process and strongly depends on heating rate and final temperature. At $80^{\circ} \mathrm{C} \mathrm{s}^{-1}$ and $800{ }^{\circ} \mathrm{C}$, for instance, only $3 \mathrm{~s}$ are needed to completely remove $\mathrm{BaCO}_{3}$ and to grow a $300 \mathrm{~nm}$ thick YBCO film (Fig. 2d, e). In some particular cases, homogeneously nucleated YBCO nanocrystals can eventually recrystallize, as suggested by synchrotron XRD analysis (Supplementary Figure 2a).

We conclude that TLAG-CSD is an ultrafast non-equilibrium and versatile process to grow epitaxial YBCO films at $c$ axis growth rates of $\sim 100 \mathrm{~nm} \mathrm{~s}^{-1}$ from different routes. This is $~ 10-100$ times faster than most of the thin film processes presently used ${ }^{8,29-32}$ to grow coated conductors and it confirms fast growth rates observed in other liquid-assisted techniques, such as in REBCO single crystal growth, REBCO bulk ceramic melt-textured growth ${ }^{33}$ or REBCO films ${ }^{14}$. However here, the process is not restricted to the use of an equilibrium liquid phase and we demonstrate that the transient liquid formation is compatible with high-throughput CSD film manufacturing.

TLAG-CSD film properties. Optimized conditions for the TLAG-CSD process gives rise to highly epitaxial YBCO films of 200-300 nm thickness (out-of-plane and in-plane textures of $\Delta \omega$ $<0.3^{\circ}$ and $\Delta \varphi<0.5^{\circ}$, respectively, Supplementary Figure 4$)$. Also, very high critical current densities of $5 \mathrm{MA} \mathrm{cm}^{-2}$ at $77 \mathrm{~K}$ and 39 $\mathrm{MA} \mathrm{cm} \mathrm{cm}^{-2}$ at $5 \mathrm{~K}$ with transition temperature $\sim 90 \mathrm{~K}$ are reached (Fig. 3 and Supplementary Figure 5) upon tuning the process parameters $\left(P_{\text {total }}, P_{\mathrm{O} 2}, T\right.$, ramp rates, substrate). The particular case shown in Fig. 3 is for a $90 \mathrm{~nm}$ thick YBCO film using the $\mathrm{P}_{\mathrm{O} 2}$-route with the reaction mechanism of Eq. $(2,3)$.

Liquid-assisted growth, however, exposes some challenges that need to be properly addressed for each type of route. Coarsening of unreacted precursor phases in the liquid, liquid reactivity with the substrates (or the buffer layers) owing to its highly corrosive nature and, improper substrates wettability with the viscous liquid ${ }^{34}$ or trapped liquid at grain boundaries, are some of them. Nevertheless, we have proved that superconducting films with very high critical current densities can be grown if the 


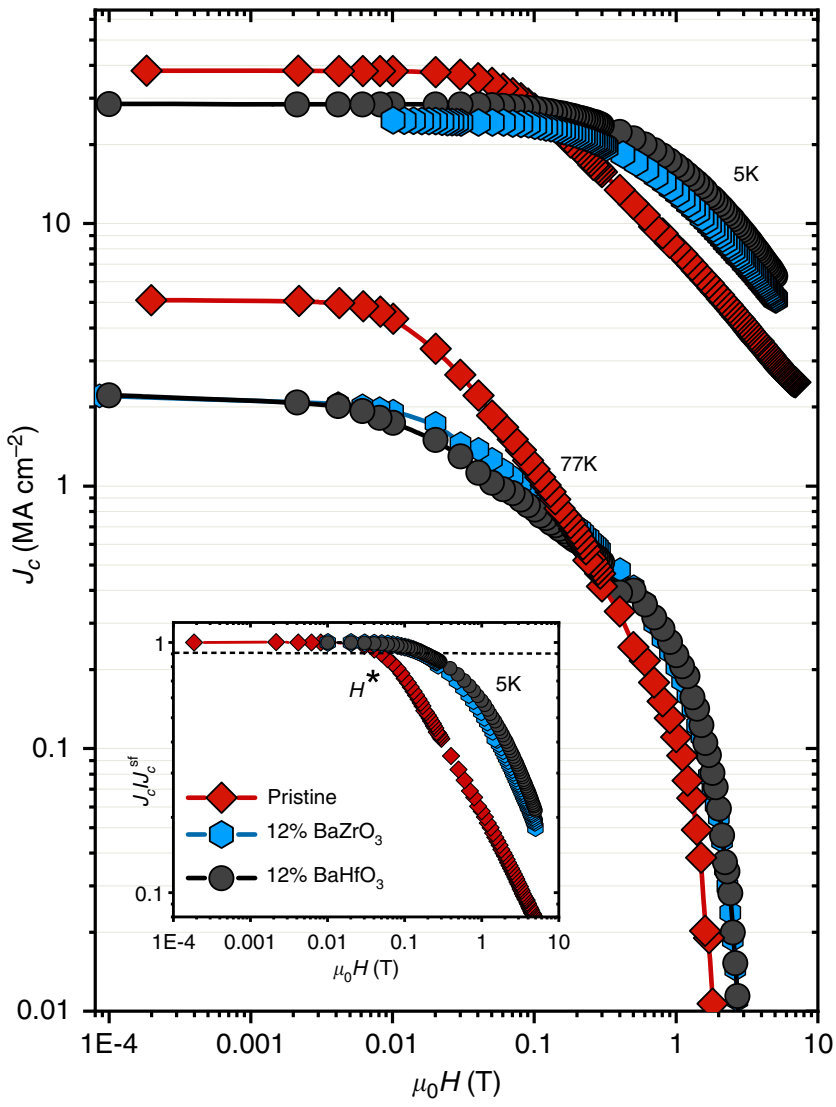

Fig. 3 Inductive critical current density, $\boldsymbol{J}_{\mathbf{c}}$ as a function of the magnetic field for TLAG films. A pristine YBCO film and two nanocomposites of $12 \%$ molar $\mathrm{BZO}$ and $12 \%$ molar $\mathrm{BHO}$ of composition 3:7 are shown for $5 \mathrm{~K}$ and $77 \mathrm{~K}$. The growth conditions followed the $\mathrm{P}_{\mathrm{O} 2}$-route at temperature of $835^{\circ} \mathrm{C}$ and $P_{\mathrm{O} 2 \text {,final }}=10^{-3}$ bar. Samples are $90 \mathrm{~nm}$ thick. The inset shows the normalized $J_{C}$ for the self-field value at $5 \mathrm{~K}$ for the same three films.

The $\mathrm{H}^{\star}$ parameter is defined with the criterion of the value of $\mathrm{H}$ at 0.9 times $J_{c}$ at self-field. The irreversibility line obtained from electrical transport measurements is shown in Supplementary Figure 6, displaying an $\mu_{0} \mathrm{H}_{\text {irr }}(77 \mathrm{~K}) \sim 8.1 \mathrm{~T}$.

aforementioned obstacles are overcome, in agreement with previous liquid-assisted CCs growth ${ }^{14,25}$.

Figure 4 shows a cross-section STEM image demonstrating the quality of the film growth. Each route has a distinctive microstructure. However, the main defects are the $\mathrm{Y}_{2} \mathrm{Ba}_{4} \mathrm{Cu}_{8} \mathrm{O}_{\mathrm{x}}$ (Y248) intergrowths (double $\mathrm{CuO}$ chains), similarly to the TFACSD process ${ }^{35}$. For the T-route, at low heating rates of $0.4^{\circ} \mathrm{C} \mathrm{s}^{-1}$ a microstructure with only few Y248 intergrowth (indicated by yellow arrows) is observed (Fig. 4a), in agreement with the low growth rate identified at low temperatures, where most of the film would grow. At increased heating rates of $20^{\circ} \mathrm{C} \mathrm{s}^{-1}$, we observe a high density of defects, mainly double and triple short $\mathrm{CuO}$ chain intergrowths (also marked with yellow arrows) and nanoscale ab grains in the range of $5-10 \mathrm{~nm}$ distributed in the bulk of the film (Fig. 4b). This is in agreement with the ultrafast TLAG-CSD growth process achieved at high temperatures. Overall, we want to highlight that different microstructures are reached depending on the process route and parameters, making TLAG-CSD a very tuneable process for vortex pinning.

Growth of TLAG nanocomposites. The growth of nanocomposites has demonstrated to be the path to pin vortices and reach high critical currents at high magnetic fields in coated
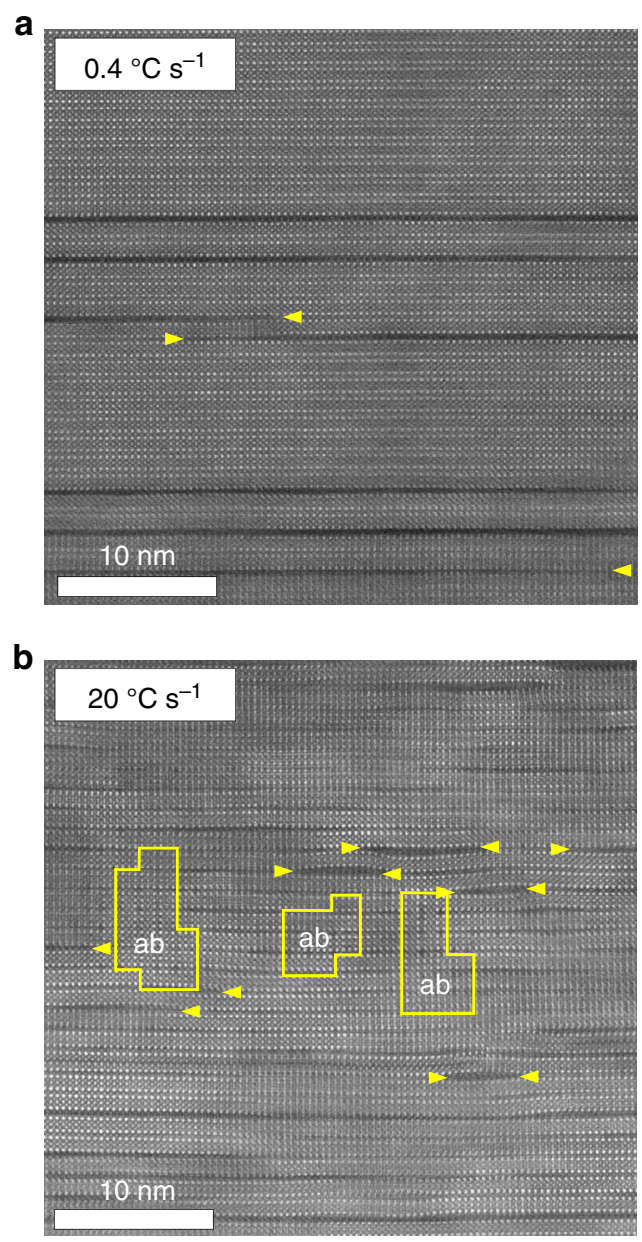

Fig. 4 STEM cross-section images of TLAG-YBCO films highlighting the differences in microstructure for the T-route. a at a heating rate of $0.4^{\circ} \mathrm{C}$ $\mathrm{s}^{-1}$, where highly epitaxial film with a low density of defects (Y248 intergrowth) is shown, and $\mathbf{b}$ at $20^{\circ} \mathrm{C} \mathrm{s}^{-1}$ heating rate, that shows a highly distorted YBCO matrix with a high density of short Y248 intergrowths and nanoscale ab grains. Yellow arrows indicate some of the Y248 intergrowths.

conductors, especially for large magnet applications ${ }^{4-7,36,37}$. In CSD, most of the effort has been done on adding metalorganic salts to the precursor solution; a method that relies on the spontaneous segregation process of nanoparticles during $\mathrm{YBCO}$ growth ${ }^{37,38}$. However, this approach is rather complex with TLAG-CSD, as one would need to finely control two ultrafast quasi-simultaneous nucleation processes. Instead, we demonstrate that TLAG-CSD is compatible with nanocomposites growth when preformed nanoparticles are stabilized in the precursor solution. This result represents a second breakthrough for the TLAG-CSD process, beyond the ultrafast growth. In this case, a colloidal solution of $\mathrm{BaMO}_{3}(\mathrm{BMO}, M=\mathrm{Zr}$, Hf) nanoparticles of $5 \mathrm{~nm}$ in diameter (Fig. 5a) was stabilized in the $\mathrm{Y}, \mathrm{Ba}, \mathrm{Cu}$ propionates-based metalorganic solution, in a similar way as for TFA-CSD ${ }^{39}$. Cross-section STEM images of a nanocomposite

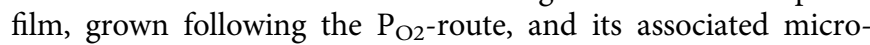
structure are shown in Fig. 5b. Notice that the final nanoparticles size, identified by STEM measurements, is in the range of $6-10 \mathrm{~nm}$. These values have also been confirmed by XRD crystallite size analysis, evidencing a low degree of coarsening. Nanocomposites with large nanoparticle content (up to $32 \% \mathrm{~mol}$ ) have been prepared maintaining high $T_{\mathrm{c}}$ in the range of $88-92 \mathrm{~K}$ and epitaxial growth (Supplementary Figure 4 and 5). The 


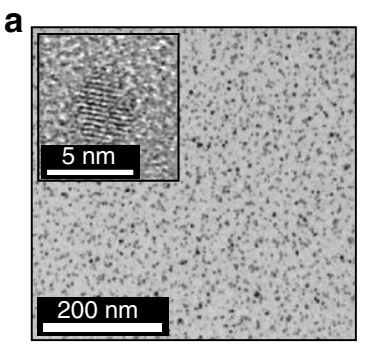

C

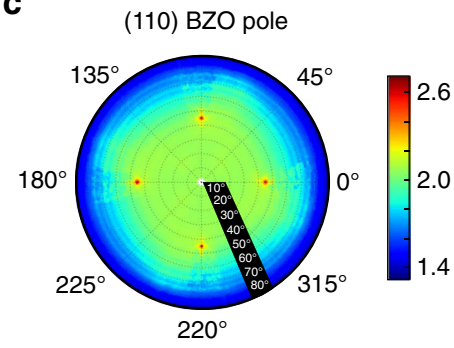

b

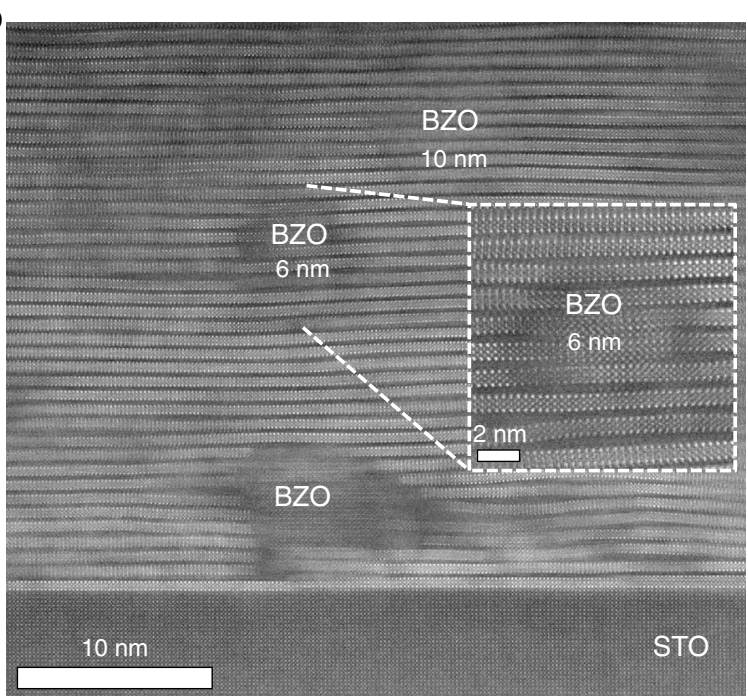

Fig. 5 Growth of TLAG nanocomposites. a TEM image of $5 \mathrm{~nm} \mathrm{BaHfO}$ nanoparticles in the colloidal solution. b STEM image of a $12 \%$ Molar $\mathrm{BaZrO}_{3}$ (BZO) nanocomposite (in a $90 \mathrm{~nm}$ thick $\mathrm{YBCO}$ layer) on $\mathrm{SrTiO}_{3}$ single crystal substrate, where few BZO nanoparticles are identified. The inset shows a high resolution image of the region with a $6 \mathrm{~nm} B Z O$ nanoparticle embedded in the YBCO matrix; strong lattice distortions and Y248 intergrowth generated are evidenced. c (110) Pole figure of the $24 \%$ molar BZO nanocomposite where epitaxial orientation of the nanoparticles is demonstrated (data are fourfold symmetrized, XRD analysis performed at SOLEIL Synchrotron).

self-field critical current density starts to decrease for nanocomposites with nanoparticles contents beyond $24 \%$ mol, which can be explained by some agglomeration of the nanoparticles and minor non-oriented grains.

A remarkable feature of TLAG-CSD nanocomposites is that BMO nanoparticles tend to grow epitaxially with the YBCO matrix (see (110) pole-figure of BZO in Fig. 5c). This effect is similar to that observed in techniques based on simultaneous deposition and growth (PLD, MOCVD) $)^{36,40}$ and it is opposite to the TFA-CSD route where nanoparticles are found randomly oriented $^{7,37}$. Quantification of the epitaxial fraction following the method reported in ${ }^{7}$ gives an epitaxial fraction in the range from 85 to $95 \%$ for the TLAG-CSD nanocomposites. This demonstrates that the liquid phase allows nanoparticles rotation inside the liquid to minimize interfacial energies. This rotation actually occurs simultaneously to an enhanced crystallinity and some recrystallization of the $\mathrm{BMO}$ nanoparticles (Fig. 5b). On the contrary, in the TFA-CSD process, the solid-state hinders the rotation of the nanoparticles, i.e., nanoparticles remain randomly oriented, even if some recrystallization leading to faceted interfaces was also observed ${ }^{7}$.

We also point out that the density of Y248 intergrowths is extremely high in TLAG nanocomposites (Fig. 5b). This indicates that the driving force for Y248 formation in this process is not provided by the minimization of large incoherent interfaces like in the TFA-CSD case ${ }^{7}$. Instead, we propose that the highly defective YBCO matrix is induced by the ultrafast growth of the nanocomposite film. It is known that the Y248 intergrowth is one of the lowest energy defects able to accommodate large distortions in $\mathrm{YBCO}^{35}$. The high density of local nanoscale distortions can be characterized by XRD Williamson-Hall analysis in terms of nanostrain ${ }^{7}$. The corresponding increase of the nanostrain with the density of nanoparticles (Supplementary Figure 5) is in agreement with previous analysis on TFA-CSD nanocomposites, where local strained regions serve as strong artificial pinning centers due to strain induced Cooper-pair suppression ${ }^{7}$.

Figure 3 illustrates the effect of nanoparticles and associated microstructure on the magnetic field dependence of the critical current density, $J_{\mathrm{c}}$, for a $12 \% \mathrm{~mol}$ of $\mathrm{BZO}$ and $12 \% \mathrm{~mol} \mathrm{BHO}$ nanocomposite films of $90 \mathrm{~nm}$ thickness. Notice that the critical current density of TLAG-CSD nanocomposites out-performs that of pristine samples already above a magnetic field of $0.1-0.2 \mathrm{~T}$, even though the self-field values were not properly optimized yet. The much smoother in-field $J_{c}$ dependence remains for the whole-temperature range and the crossover field identified by the $H^{\star}$ parameter (see Fig. 4 inset) can even reach values of $200 \mathrm{mT}$, which are on the order of the highest results measured by SQUID in CSD YBCO nanocomposite films ${ }^{39}$. For the same values of nanostrain and therefore of local distortion, nanocomposites present a large increase of $H^{\star}$ with respect to pristine samples (Supplementary Figure 5), showing that there is another pinning mechanism acting beyond the nanostrain effect. We suggest that nanoparticles of 6-8 $\mathrm{nm}$ (diameter 2-3 times higher than the vortex coherence length, $\xi_{\mathrm{ab}}$ ) could be directly pinning vortices ${ }^{41}$, thus the aforementioned $H^{\star}$ enhancement in TLAG nanocomposites might be partially governed by nanoparticles acting themselves as core pinning centers. Such a scenario might pave the way to radical performance increase in CSD nanocomposite films upon continuing nanoparticle miniaturization and the prevention of coarsening at high concentrations. We emphasize that the fast crystallization kinetics of the TLAG approach can advantageously be used to push the frontier of nanocomposite-coated conductor manufacturing.

\section{Discussion}

We have developed a non-equilibrium process to grow YBCO films and nanocomposites at ultrafast growth rates $\left(\sim 100 \mathrm{~nm} \mathrm{~s}^{-1}\right)$, which can be combined with low-cost CSD and large area ink jet printing deposition techniques. The breakthrough idea is the use of a transient liquid to grow epitaxial YBCO films. Critical current densities of $5 \mathrm{MA} \mathrm{cm}^{-2}$ at $77 \mathrm{~K}$ and in-field enhanced properties for nanocomposites based on colloidal nanoparticles solutions have been demonstrated. This growth technique appears very promising for high-throughput preparation of YBCO films and if demonstrated for long length thick coated conductors, it could widely spread the use of high-temperature superconducting materials for practical applications. Preliminary work on growth of $1 \mu \mathrm{m}$ thick TLAG-CSD films show no difficulties in eliminating $\mathrm{BaCO}_{3}$ in any of the routes here reported, as well as no reactivity on $\mathrm{LaMnO}_{3}$-buffered coated conductors. In our opinion, this suggests a great future for this methodology, easily scale-up and integrated in simple reel-to-reel furnaces, to create a highthroughput and low-cost CC manufacturing schemes for fabrication of coated conductors. It is based on very general kinetic and thermodynamic principles and could therefore be applied to grow other functional nanomaterials. 


\section{Methods}

Sample preparation. Epitaxial $c$ axis-oriented $\mathrm{YBCO}$ and $\mathrm{BMO}(M=\mathrm{Zr}, \mathrm{Hf})$ nanocomposites thin films have been grown on single-crystalline (100) $\mathrm{SrTiO}_{3}$ substrates. Samples were prepared from metalorganic precursor solutions with two stoichiometries: $\mathrm{YBa}_{2} \mathrm{Cu}_{3} \mathrm{O}_{\mathrm{x}}$ and $\mathrm{YBa}_{2} \mathrm{Cu}_{4.66} \mathrm{O}_{\mathrm{x}} . \mathrm{Y}, \mathrm{Ba}$, and $\mathrm{Cu}$ anhydrous acetates were dissolved in propionic acid for four hours at $50{ }^{\circ} \mathrm{C}$ and afterwards anhydrous methanol (in a 50/50) mixer was added to reach a $1 \mathrm{M}-1.5 \mathrm{M}$ concentration depending on desired film thickness. In some cases, a $5 \% \%_{\mathrm{v} / \mathrm{v}}$ triethanolamine was also added to reach larger thickness. Films of thickness from $90 \mathrm{~nm}$ up to $500 \mathrm{~nm}$ were studied. Spin coating was used for films up to $300 \mathrm{~nm}$ thickness with a pyrolysis of $500{ }^{\circ} \mathrm{C}$ for $5 \mathrm{~min}$ in an oxygen atmosphere, whereas a home-made ink jet printer with microfab piezoelectric nozzles was used for film thickness from 300 up to $500 \mathrm{~nm}$. Superconducting YBCO films were grown in a Rapid Thermal Annealing furnace from Annealsys AS.Micro equipped with a $\mathrm{SiC}$ coated graphite susceptor, capable of reaching heating rates up to $80^{\circ} \mathrm{C} \mathrm{s}^{-1}$ with calibrated temperatures. The T-route or $\mathrm{P}_{\mathrm{O} 2}$-route explained in the main text were used as convenient. In the T-route, temperature was increased up to a range between 650 and $850^{\circ} \mathrm{C}$ at a heating rate between $0.4^{\circ} \mathrm{C} \mathrm{s}^{-1}$ and $80^{\circ} \mathrm{C} \mathrm{s}^{-1}$, at constant oxygen partial pressure between $5 \times 10^{-4}$ and $10^{-2}$ bar (as shown in Fig. 1). Best results were obtained around $830^{\circ} \mathrm{C}$ at $10^{-3}$ bar for a heating rate of $20^{\circ} \mathrm{Cs}^{-1}$. In the $\mathrm{P}_{\mathrm{O} 2}$-route, the film was heated up to the desired temperature (between $750^{\circ} \mathrm{C}$ and $850^{\circ} \mathrm{C}$ ) at a heating rate between $0.4^{\circ} \mathrm{C} \mathrm{s}^{-1}$ and $20^{\circ} \mathrm{C} \mathrm{s}^{-1}$ at an oxygen partial pressure between $2 \times 10^{-5}$ and $10^{-6} \mathrm{bar}$, and subsequently $P_{\mathrm{O} 2}$ was fast $(<1 \mathrm{~s})$ increased up to the range of $10^{-4}-10^{-2}$ bar. Best results were obtained heating the film up to $835^{\circ} \mathrm{C}$ at $20^{\circ} \mathrm{C} \mathrm{s}^{-1}$ at $10^{-5}$ bar with a fast change of $P_{\mathrm{O} 2}$ up to $10^{-3}$ bar. The total pressure was also varied from $10^{-2}$ to 1 bar depending on the experiment and route used. Films were subsequently oxygenated in a tubular furnace at $450^{\circ} \mathrm{C}$ for $200 \mathrm{~min}$ in oxygen flowing atmosphere. $\mathrm{BaMO}_{3}(M=$ $\mathrm{Zr}$, Hf) nanoparticles were prepared following a polyol solvothermal method by mixing $\mathrm{Zr}$ or Hf alkoxide precursors and Barium Hydroxide into a mixture of alcoholic solvents ${ }^{39}$. This mixture was treated in an autoclave at $180{ }^{\circ} \mathrm{C}$ for $60 \mathrm{~min}$ to achieve the crystallization of the nanoparticles. $\mathrm{BaZrO}_{3}(\mathrm{BZO})$ of $5 \mathrm{~nm}$ of diameter and $\mathrm{BaHfO}_{3}(\mathrm{BHO})$ of $4 \mathrm{~nm}$ in diameter were obtained in stable colloidal suspensions of 100-120 mM of concentration in ethanol. Afterwards, the colloidal BMO solutions were stabilized in the YBCO precursor solutions to reach the desired final concentration of metal salts with different molar percentages of nanoparticles (up to $32 \% \mathrm{~mol}$ ). All solutions were characterized by water content, contact angle, viscosity, metal-stoichiometry and dynamic light scattering (DLS)

The eutectic line of Fig. $1 \mathrm{~b}$ was determined from data on $3 \mathrm{BaO}-7 \mathrm{CuO}$ binary pyrolyzed powders and thin films on $\mathrm{MgO}$ substrates. They were analyzed by thermogramivetry and differential thermal analysis using the Setsys evolution apparatus of Setaram, at subatmospheric conditions $P_{\text {total }}=P_{\mathrm{O} 2}$ and at a heating rate of $5^{\circ} \mathrm{C} \mathrm{min}^{-1}$.

XRD. The phase and texture analysis of the grown films and nanocomposites was analyzed with a high resolution Discover D8 Bruker diffractometer (X-ray energy $=8.049 \mathrm{keV}$ ) equipped with a Lynxeye XE energy-dispersive $1-\mathrm{D}$ detector. The quantitative determination of epitaxial fraction of nanoparticles was investigated with a Bruker-AXS D8 Advance diffractometer operating with $\mathrm{Cu} K_{\alpha}$ radiation equipped with a General Area Detector Diffraction System. Non-uniform r.m.s. strain (nanostrain) was determined from XRD integral-breadth measurements through semiquantitative Williamson-Hall plots using the Discover D8 Bruker system. Debye Scherrer equation was used to determine the nanoparticles size from the (200) reflection.

In situ XRD synchrotron analysis were carried out at DiffAbs beamline at SOLEIL synchrotron with a beam energy of $18 \mathrm{keV}$, heating ramps of $5^{\circ} \mathrm{C} \mathrm{s}^{-1}$ and 1 bar of total pressure. An area detector (X-ray hybrid pixel area detector, XPAD) was used ${ }^{42}$ with acquisition times per data point of $500 \mathrm{~ms}$ (for phase evolution before YBCO growth) and $100 \mathrm{~ms}$ (for YBCO growth), respectively. To follow random phases, grazing XRD scans were recorded, whereas to follow YBCO growth the Bragg conditions with the (005) peak were met $(\theta-2 \theta$ geometry). The experiments were run in a DHS 1100 Anton Paar heater covered with a graphite dome, with maximum heating rates of $5^{\circ} \mathrm{C} \mathrm{s}^{-1}$ and equipped with a double connection to the vacuum pumps and to the gas inlet system. Electrovalves were used to modulate the total pressure, while an oxygen sensor was used to pre-set the $P_{\mathrm{O} 2}$. For $\mathrm{P}_{\mathrm{O} 2}$-route and T-route, different combinations of $\mathrm{N}_{2}$ and Air gases were mixed with a mass flow controller to meet the required conditions. Pole figures (characteristics of the nanoparticles embedded in the YBCO matrix) were recorded during synchrotron experiments at DiffAbs using the XPAD area detector.

Transmission electron microscopy. In addition, the nanoparticle size in the colloidal solutions were determined by TEM studies with a JEOL of $120 \mathrm{kV} 1210$ TEM, with a resolution point of $3.2 \AA$ and DLS Zetasizer Nano Zs with measurement range of $0.3 \mathrm{~nm}-10.0 \mu \mathrm{m}$ and sensitivity of $0.1 \mathrm{mg} \mathrm{mL}^{-1}$. The microstructure, atomic defect structure of films and nanocomposites, and nanoparticles size in the nanocomposite films were studied with a STEM using a FEI Tecnai F20 S/TEM operated in TEM mode at $200 \mathrm{kV}$, and with a X-FEG gun, a CETCOR probe corrector and a Gatan TRIDIEM 866 ERS energy filter operated in STEM mode at $300 \mathrm{kV}$. Specimens for STEM were prepared by conventional methods, by grinding, dimpling and Ar ion milling or Focused Ion Beam (FIB). A FIB Zeiss 1560XB Cross Beam was also used to perform and visualize the cross-section of Supplementary Figure 1a.
Physical properties. Inductive critical current densities were obtained by applying the Bean critical-state model to thin films. The hysteretic magnetization curves were measured with a commercial superconducting quantum interference device (SQUID) magnetometer from Quantum Design equipped with a superconducting magnet of $7 \mathrm{~T}$. The $H^{*}$ parameter was determined from the $\mathrm{J}_{c}(\mathrm{H})$ curves with a criterion of $0.9 \times J_{c}$ at self-field at $5 \mathrm{~K}$. Critical temperature measurements were determined using a PPMS Quantum Design system using the Van der Pauw method and the derivative criterion. The irreversibility line was measured through a bridge of $40 \mu \mathrm{m}$ in the same PPMS system using a criterion of $\mathrm{R}\left(\mathrm{T}_{\mathrm{irr}}\right) / \mathrm{R}(95 \mathrm{~K})=$ 0.001 . Film thickness was determined from a three-dimensional profilometer P16 from KLA-Tencor.

\section{Data availability}

The data that support the findings of this study are available from the corresponding authors on reasonable request.

Received: 11 October 2019; Accepted: 29 November 2019; Published online: 17 January 2020

\section{References}

1. Larbalestier, D., Gurevich, A., Feldmann, D. M. \& Polyanskii, A. High-T superconducting materials for electric power applications. Nature 414, 368-377 (2001).

2. Obradors, X. \& Puig, T. Coated conductors for power applications: materials challenges. Supercond. Sci. Technol. 27, 044003-044017 (2014).

3. Marchionini, B. G., Yamada, Y., Martini, L. \& Ohsaki, H. High-temperature superconductivity: a roadmap for electric power sector applicationh-temperature superconductivity: a roadmap for electric power sector applications, 2015-2030. IEEE Trans. Appl. Supercon. 27, $0500907-0500907$ (2017)

4. Haugan, T., Barnes, P. N., Wheeler, R., Meisenkothen, F. \& Sumption, M. Addition of nanoparticle dispersions to enhance flux pinning of the $\mathrm{YBa}_{2} \mathrm{Cu}_{3} \mathrm{O}_{7-\mathrm{x}}$ superconductor. Nature 430, 867-870 (2004).

5. MacManus-Driscoll, J. L. et al. Strongly enhanced current densities in superconducting coated conductors of $\mathrm{YBa}_{2} \mathrm{Cu}_{3} \mathrm{O}_{7-\mathrm{x}}+\mathrm{BaZrO}_{3}$. Nat. Mater. 3, 439-443 (2004)

6. Kang, $S$. et al. High-performance high- $\mathrm{T}_{\mathrm{c}}$ superconducting wires. Science 311, 1911-1914 (2006)

7. Llordés, A. et al. Nanoscale strain-induced pair suppression as a vortexpinning mechanism in high-temperature superconductors. Nat. Mater. 11, 329-336 (2012)

8. Solovyov, V., Dimitrov, I. K. \& Li, Q. Growth of thick $\mathrm{YBa}_{2} \mathrm{Cu}_{3} \mathrm{O}_{7}$ layers via a barium fluoride process. Supercond. Sci. Technol. 26, 013001-013019 (2013).

9. Yun, K. S. et al. Vapor-liquid-solid tri-phase pulsed-laser epitaxy of $\mathrm{RBa}_{2} \mathrm{Cu}_{3} \mathrm{O}_{7-y}$ single-crystal films. Appl. Phys. Lett. 80, 61-63 (2002).

10. Ohnishi, T., Huh, J. U., Hammond, R. H. \& Jo, W. High rate in situ $\mathrm{YBa}_{2} \mathrm{Cu}_{3} \mathrm{O}_{7}$ film growth assisted by liquid phase. J. Mater. Res. 19, 977-981 (2004).

11. Kursumovic, A. et al. Hybrid liquid phase epitaxy processes for $\mathrm{YBa}_{2} \mathrm{Cu}_{3} \mathrm{O}_{7}$ film growth. Supercond. Sci. Technol. 17, 1215-1223 (2004).

12. Scheel, H. J. Liquid Phase Epitaxy of Electronic, Optical and Optoelectronic Materials, Ed. P. Capper and M. Mauk, John Wiley \& Sons, (2007).

13. Ichino, Y. et al. Possibility of high deposition rate in $\mathrm{SmBa}_{2} \mathrm{Cu}_{3} \mathrm{O}_{\mathrm{y}}$ films prepared using the Vapor-Liquid-Solid growth mode. Jpn. J. Appl Phys. 45, $758-760$ (2006).

14. Lee, J. et al. RCE-DR, A novel process for coated conductor fabrication with high performance. Supercond. Sci. Technol. 27, 044018-6 (2014).

15. MacManus-Driscoll, J. L. et al. Strong pinning in very fast grown reactive coevaporated $\mathrm{GdBa}_{2} \mathrm{Cu}_{3} \mathrm{O}_{7}$ coated conductors. Appl. Phys. Lett. Mater. 2, 086103 (2014).

16. Feighan, J. P. F., Kursumovic, A. \& MacManus-Driscoll, J. L. Materials design for artificial pinning centres in superconductor PLD coated conductors. Supercond. Sci. Technol. 30, 123001-123016 (2017).

17. Gamalski, A. D., Tersoff, J., Sharma, R., Ducati, C. \& Hofmann, S. Formation of metastable liquid catalyst during subeutectic growth of Germanium nanowires. Nano Lett. 10, 2972-2976 (2010).

18. Kursumovic, A., Cheng, Y., Glowacki, B., Madsen, J. \& Evetts, J. Study of the rate-limiting processes in liquid-phase epitaxy of thick $\mathrm{YBaCuO}$ films. J. Cryst. Growth 218, 45-56 (2000).

19. Chen, N., Rothman, S., Routbort, J. \& Goretta, K. Tracer diffusion of Ba and Y in $\mathrm{YBa}_{2} \mathrm{Cu}_{3} \mathrm{O}_{\mathrm{x}}$. J. Mat. Res. 7, 2308-2316 (1992).

20. Chernov, A. A. Notes on interface growth kinetics 50 years after Burton, Cabrera and Frank. J. Cryst. Growth 264, 499-518 (2004).

21. Kursumovic, A. et al. High critical current densities in $\mathrm{YBa}_{2} \mathrm{Cu}_{3} \mathrm{O}_{7-\mathrm{x}}$ films grown at high rates by hybrid liquid phase epitaxy. Appl. Phys. Lett. 87, 252507-3 (2005). 
22. Klemenz, C. \& Scheel, H. J. Solubility of $\mathrm{YBCO}$ and $\mathrm{NdBCO}$ the $\mathrm{BaO} / \mathrm{CuO}$ flux. J. Cryst. Growth 200, 435-440 (1999).

23. Krauns, C., Sumida, M., Tagami, M., Yamada, Y. \& Shiohara, Y. Solubility of $\mathrm{RE}$ elements into $\mathrm{Ba}-\mathrm{Cu}-\mathrm{O}$ melts and the enthalpy of dissolution. Z. für. Phys. B Condens. Matter 96, 207-212 (1994).

24. Yao, X. \& Shiohara, Y. Process for high growth rate and high superconducting properties of REBCO single crystals. Mater. Sci. Eng. B 53, 11-17 (1998).

25. MacManus-Driscoll, J. L. et al. $\mathrm{YBa}_{2} \mathrm{Cu}_{3} \mathrm{O}_{7}$ coated conductor growth by Hybrid Liquid Phase Epitaxy. IEEE Trans. Appl. Superconductivity 17, 2537-2541 (2007).

26. Vilardell, M. et al. Flexible manufacturing of functional ceramic coatings by inkjet printing. Thin Solid Films 548, 489-497 (2013).

27. Lindemer, T. B. \& Specht, E. D. The $\mathrm{BaOCuO}$ system. Solid-liquid equilibria and thermodynamics of $\mathrm{BaCuO}_{2}$ and $\mathrm{BaCu}_{2} \mathrm{O}_{2}$. Phys. C. 255, 81-94 (1995).

28. MacManus-Driscoll, J. L., Bravman, J. C. \& Beyers, R. B. Phase-equilibria in the $\mathrm{Y}-\mathrm{Ba}-\mathrm{Cu}-\mathrm{O}$ system and melt processing of $\mathrm{Ag}$ clad $\mathrm{YBa}_{2} \mathrm{Cu}_{3} \mathrm{O}_{7-\mathrm{x}}$ tapes at reduced oxygen partial pressure. Phys. C. 241, 401-413 (1995).

29. Pahlke, P. et al. Influence of artificial pinning centers on structural and superconducting properties of thick YBCO films on ABAD-YSZ templates. Supercond. Sci. Technol. 31, 004007-004009 (2018).

30. Chepikov, V. et al. Introduction of $\mathrm{BaSnO}_{3}$ and $\mathrm{BaZrO}_{3}$ artificial pinning centres into $2 \mathrm{G}$ HTS wires based on PLD-GdBCO films. Phase I of the industrial R\&D programme at SuperOx. Supercond. Sci. Technol. 30, 124001-124012 (2017).

31. Abraimov, D. et al. Double disordered YBCO coated conductors of industrial scale: high currents in high magnetic field. Supercond. Sci. Technol. 28, 114007-114008 (2015).

32. Majkic, G. et al. Engineering current density over $5 \mathrm{kAmm}^{-2}$ at $4.2 \mathrm{~K}, 14 \mathrm{~T}$ in thick film REBCO tapes. Supercond. Sci. Technol. 31, 10LT01-16LT01 (2018).

33. Shiohara, Y. \& Endo, A. Crystal growth of bulk high- $\mathrm{T}_{\mathrm{c}}$ superconducting oxide materials. Mat. Sci. Eng. R19, 1-86 (1997).

34. Krauns, Ch, Tagami, M., Yamada, Y., Nakamura, M. \& Shiohara, Y. Wetting between prospective crucible materials and the $\mathrm{Ba}-\mathrm{Cu}-\mathrm{O}$ melt. J. Mater. Res $\mathbf{9}$, 1513-1518 (1994).

35. Guzman, R. et al. Probing localized strain in solution-derived $\mathrm{YBa}_{2} \mathrm{Cu}_{3} \mathrm{O}_{7-\delta}$ nanocomposite thin films. Phys. Rev. Mat. 1, 024801-024807 (2017).

36. $\mathrm{Xu}, \mathrm{A}$. et al. Strongly enhanced vortex pinning from 4 to $77 \mathrm{~K}$ in magnetic fields up to $31 \mathrm{~T}$ in $15 \mathrm{~mol} . \% \mathrm{Zr}$-added (Gd, Y)-Ba-Cu-O superconducting tapes. Appl. Phys. Lett. Mater. 2, 046111-046118 (2014).

37. Miura, M. et al. Tuning nanoparticle size for enhanced functionality in perovskite thin films deposited by metal organic deposition. NPG Asia Mater. 9, e447 (2017).

38. Gutierrez, J. et al. Strong isotropic flux pinning in $\mathrm{YBa}_{2} \mathrm{Cu}_{3} \mathrm{O}_{7-\mathrm{x}}-\mathrm{BaZrO}_{3}$ nanocomposite superconductor films derived from chemical solutions. Nat. Mater. 6, 367-373 (2007).

39. Obradors, $\mathrm{X}$. et al. Epitaxial $\mathrm{YBa}_{2} \mathrm{Cu}_{3} \mathrm{O}_{7-\mathrm{x}}$ nanocomposite films and coated conductors from $\mathrm{BaMO}_{3}(\mathrm{M}=\mathrm{Zr}, \mathrm{Hf})$ colloidal solutions. Supercond. Sci. Technol. 31, 044001-044016 (2018).

40. Cantoni, C. et al. Strain-driven oxygen deficiency in self-assembled, nanostructured, composite oxide films. ACS Nano 6, 4783-4789 (2011)

41. Koshelev, A., Sadovskyy, I., Phillips, C. \& Glatz, A. Optimization of vortex pinning by nanoparticles using simulations of the time-dependent GinzburgLandau model. Phys. Rev. B 93, 060508-5 (2016).

42. Mocuta, C. et al. Fast pole figure acquisition using area detectors at the DiffAbs beamline - Synchrotron SOLEIL. J. Appl. Cryst. 46, 1842-1853 (2013).

\section{Acknowledgements}

Authors acknowledge EU for ULTRASUPERTAPE ERC-2014-ADG-669504; COACHSUPENERGY MAT2014-51778-C2-1-R and MAT2014-51778-C2-2-R from MINECO; SuMaTe RTI2018-095853-B-C21 and RTI2018-095853-B-C22 from
MICINN and co-financing by the European Regional Development Fund; 2017-SGR 1519 from Generalitat de Catalunya; and EU COST Action NANOCOHYBRI (CA16218). ICMAB authors acknowledge the Center of Excellence award Severo Ochoa SEV-2015-0496. Authors thank Dr. Cornelia Pop, Dr. Bohores Villarejo, Dr. Bernat Mundet, and Dr Anna Palau for contributions at the initial stages of the project. We thank Diana García, Lavinia Saltarelli, Adria Pacheco, Dr. Max Sieger, and Dr. Albert Queralto for help in the feasibility study and preliminary results on thick films and coated conductors. Authors acknowledge support from SuNAM Co Ltd. for providing the buffered metallic substrates for the initial tests on coated conductors. We also acknowledge the Scientific Services at ICMAB, ICTS of IMB-CNM-CSIC, ICN2 Electron Microscopy Division and LMA-INA from Aragon, and SOLEIL Synchrotron for granting beamtime for XRD experiments and the DiffAbs beamline personnel for help. L.S. and J.J. also acknowledge their FPU-MINECO PhD grant, S.R. and N.C. thank University of Girona and Generalitat de Catalunya fort their PhD FI grant, respectively.

\section{Author contributions}

X.O. and T.P. conceived the idea and the project plan. L.S, J.J., J.B., and S.Ra. synthesized and characterized the films under the supervision of J.F., P.R., S.R., X.O., and T.P. The TEM experiments were realized by R.G. who also carried out the corresponding data treatment. N.C. synthesized the nanoparticles and stabilized them in the precursor solution with the guide of R.Y. and S.Ri. The synchrotron experiments were designed and performed by T.P., J.F., C.M., X.O., J.B., L.S., J.J., and S.Ra. Data treatment was carried out by J.B., L.S., J.J., S.R. All contributed to the interpretation. P.R. determined the eutectic transition. All authors participated in the discussion and writing of the manuscript.

\section{Competing interests}

The authors declare no competing interests.

\section{Additional information}

Supplementary information is available for this paper at https://doi.org/10.1038/s41467 019-13791-1.

Correspondence and requests for materials should be addressed to T.P.

Peer review information Nature Communications thanks the anonymous reviewer(s) for their contribution to the peer review of this work

Reprints and permission information is available at http://www.nature.com/reprints

Publisher's note Springer Nature remains neutral with regard to jurisdictional claims in published maps and institutional affiliations.

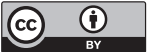

Open Access This article is licensed under a Creative Commons Attribution 4.0 International License, which permits use, sharing, adaptation, distribution and reproduction in any medium or format, as long as you give appropriate credit to the original author(s) and the source, provide a link to the Creative Commons license, and indicate if changes were made. The images or other third party material in this article are included in the article's Creative Commons license, unless indicated otherwise in a credit line to the material. If material is not included in the article's Creative Commons license and your intended use is not permitted by statutory regulation or exceeds the permitted use, you will need to obtain permission directly from the copyright holder. To view a copy of this license, visit http://creativecommons.org/ licenses/by/4.0/

(C) The Author(s) 2020 